\title{
Competencias digitales docentes: Una experiencia en el nivel Universitario
}

Digital Teaching Skills: An Experience At The University Level

\author{
Mercedes Leticia Sánchez Ambriz \\ https://orcid.org/0000-0002-2950-3782 \\ Universidad del Valle de México \\ Lidia Fabián Acevedo 2 \\ https://orcid.org/0000-0002-3577-3404 \\ Instituto Tecnológico y de Estudios Superiores de Monterrey, México \\ Dulce María Melgoza Mendoza ${ }^{3}$ \\ https://orcid.org/0000-0003-1229-8046 \\ Universidad Autónoma del Estado de Hidalgo-Escuela Superior Huejutla, México
}

Recibido: 05-12-2020

Aceptado: 29-04-2021

\section{Cita Recomendada}

Sánchez, M., Fabián, L. y Melgoza, D. (2021). Competencias digitales docentes: una experiencia en el nivel universitario. Hamut'ay, 8(1), 59-66. http://dx.doi.org/10.21503/hamu.v8i1.2236

\section{Resumen}

La emergencia sanitaria que se vive actualmente en el mundo, no sólo evidenció la dificultad para acceder y usar tecnologías, sino la poca familiaridad de los docentes con ésta. La presente investigación expone el nivel de competencias del uso de las tecnologías de la información y comunicación (TIC) de los docentes de cuatro centros de nivel universitario de México. Se adoptó un enfoque descriptivo, centrado en el método de investigación cuantitativa, los resultados permiten evidenciar, la necesidad que tiene el docente de transformar su práctica lo que conlleva, a una etapa de continuo aprendizaje de nuevos enfoques donde se fusiona la tecnología con la pedagogía, a pesar de la prevalencia de las tecnologías. Como lo muestra este estudio que el $46.9 \%$ de los docentes encuestados ocasionalmente, raramente y/o nunca han creado o editado contenidos nuevos con herramientas digitales y un $7.8 \%$ nunca crea y edita contenidos nuevos con herramientas digitales.

Palabras claves: Covid-19, competencias docentes, competencias digitales, formación docente, herramientas digitales.

1. Doctora en educación, Profesor de Tiempo Completo, Universidad del Valle de México

2 Profesora de Tiempo Completo, Instituto Tecnológico y de Estudios Superiores de Monterrey.

3 Profesora de Asignatura, Universidad Autónoma del Estado de Hidalgo- Escuela Superior Huejutla. 


\begin{abstract}
The health emergency currently being experienced in the world, not only evidenced the difficulty to access and use technologies, but also the lack of familiarity of teachers with it. The present research exposes the level of competencies in the use of information and communication technologies of teachers from four university level centers in Mexico. A descriptive approach was adopted, centered on the quantitative research method, the results show the need for teachers to transform their practice, which leads to a stage of continuous learning of new approaches where technology merges with pedagogy, despite the prevalence of technologies. As this study shows, $46.9 \%$ of the surveyed teachers have occasionally, rarely and/or never created or edited new contents with digital tools and $7.8 \%$ never create and edit new contents with digital tools.
\end{abstract}

Keywords: Covid-19, teaching skills, digital skills, teacher training, digital tools

\section{Introducción}

De acuerdo con la ONU (UN, 2020a), la COVID-19 provocó el cierre de los espacios educativos y de aprendizaje, afectando al $94 \%$ de la población estudiantil mundial, sacando de la zona de confort a un gran número de docentes que consideran el desarrollo de competencias digitales como algo opcional y poco necesario; sin embargo, para dar continuidad a las clases los gobiernos decidieron transitar hacia las plataformas virtuales y transmisiones televisadas, según Schleider \& Reimers, (2020) esta situación evidenció la urgente necesidad de manejar los entornos virtuales. Por su parte, la OCDE en 2019, mencionó que antes de la crisis sanitaria los docentes tenían una fuerte necesidad de formación en el uso de tecnología y la actual crisis también evidenció la falta de fomento en éstas. De acuerdo con un informe del CONEVAL (2020), frente a esta crisis México decidió hacer uso de educación remota para poner en funcionamiento un programa de educación a distancia, que se adoptó por parte de la mayoría de las instituciones educativas; sin embargo, este paso no incluyó el saber técnico que incluye el manejo de artefactos tecnológicos, así como los procedimientos que apoyan las decisiones pedagógicas y contribuyen a responder a las exigencias de esta modalidad educativa.

Desde luego, el Gobierno de México trató de poner en marcha algunas acciones como las propuestas por la Comisión Nacional para la Mejora Continua de la Educación, de acuerdo con Mejo- redu (2020, p. 19) aquí se exhorta: ensayar y perfeccionar nuevas formas de enseñanza mediante tecnologías diversas y la necesidad de crear relaciones afectivas y pedagógicas distintas.

El reto era mayor como lo menciona Hodges et al., (2020), en muchos casos, se trató de volcar lo que se venía haciendo en el aula presencial y el resultado fue una enseñanza de emergencia en remoto, que según Ibáñez (2020) significa adaptar sus métodos en un plazo de tiempo muy corto para poder seguir impartiendo clases a todos sus estudiantes.

De esta manera, la innovación educativa siempre aplazada se vio acelerada, y una forma de hacerle frente a esta emergencia, fue organizarse entre los propios docentes, quienes vieron la pandemia como un acelerador de la transformación de la educación (Naffi, 2020), aunque el uso de las herramientas tecnológicas por sí mismas no es garantía de éxito educativo (Reich, 2020), pero estas son una estrategia para hacer que las actividades académicas permitan incentivar y motivar el aprendizaje en la virtualidad.

\section{Competencias docentes}

La irrupción de Internet y más tarde la globalización trajo consigo la ruptura de métodos de aprendizaje y la inserción de competencias en la educación refiere que se exige de una preparación del docente acorde con lo que se requiere de él (Chan y Delgado, 2009), como bien menciona Aguaded, Marín y Díaz, (2015) es un requisito indispensable para la nueva generación de docen- 
tes. Por lo tanto, las competencias docentes abarcan todo lo relacionado con la práctica educativa, con su finalidad, con la preocupación por mejorarla y con su profesionalización. En un mundo global y digitalizado, según Limón (2020), el objetivo es el aprendizaje de los estudiantes, identificar las habilidades para desarrollar y promover la mejora de las estrategias docentes que permitan enfrentar los nuevos desafíos y, en este contexto, la tecnología es parte importante del desarrollo de habilidades. Por ello, para conocer las competencias es necesario considerar tres aspectos: el contenido, la clasificación y la formación, es decir, saber qué enseñar, cómo enseñar, a quiénes se enseña y para qué, desde la perspectiva de las necesidades del desarrollo económico y social y, más concretamente, de las demandas del sistema productivo (Barnett, 2001, Álvarez, González y López 2009). Este tipo de educación hace evidente el tránsito de un proceso centrado en la enseñanza a uno centrado en el aprendizaje, lo cual supone redefinir la organización de los procesos de aprendizaje y las funciones sustantivas de las instituciones de educación superior.

En su análisis, Argudín (2001) afirma que la competencia es una convergencia de los comportamientos sociales, afectivos, cognitivos, psicológicos y sensoriales que permiten desempeñar un papel, una actividad o tarea, en el que participan tres factores constitutivos básicos en la competencia, como se muestra en la figura 1.

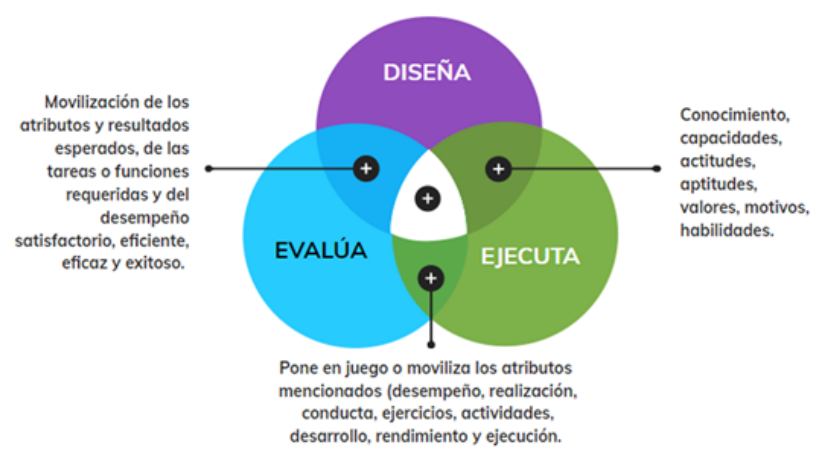

Figura 1. Factores básicos en la competencia Fuente: Elaboración propia (2020).

Por otro lado, el punto de partida para diseñar un currículo orientado hacia las competencias consiste en integrar conocimientos, potencialidades, habilidades, destrezas, prácticas y acciones de di- versa índole (personales, colectivas, afectivas, sociales, culturales) en los diferentes escenarios del aprendizaje y el desempeño (Aristimuño, 2005). La competencia, por lo tanto, es una interacción reflexiva y funcional de saberes (cognitivos, procedimentales, actitudinales y metacognitivos) enmarcada por la motivación de los estudiantes por aprender y desarrollarse como personas que puedan actuar en distintos contextos y transformar su entorno.

En cuanto al docente, parte de sus competencias profesionales es mostrar cómo se lleva a cabo la práctica educativa a partir de la reflexión de la teoría y el ejercicio de su desempeño cotidiano. Para ser eficaz, esta debe acompañarse de una actitud positiva ante las diferentes formas de aprendizaje de los estudiantes, así como de capacidades de planificación y organización de actividades significativas de acuerdo con los contenidos. Para Rivadeneira (2017), un docente preparado para los retos del presente debe mostrar las habilidades que se muestran en la figura 2.

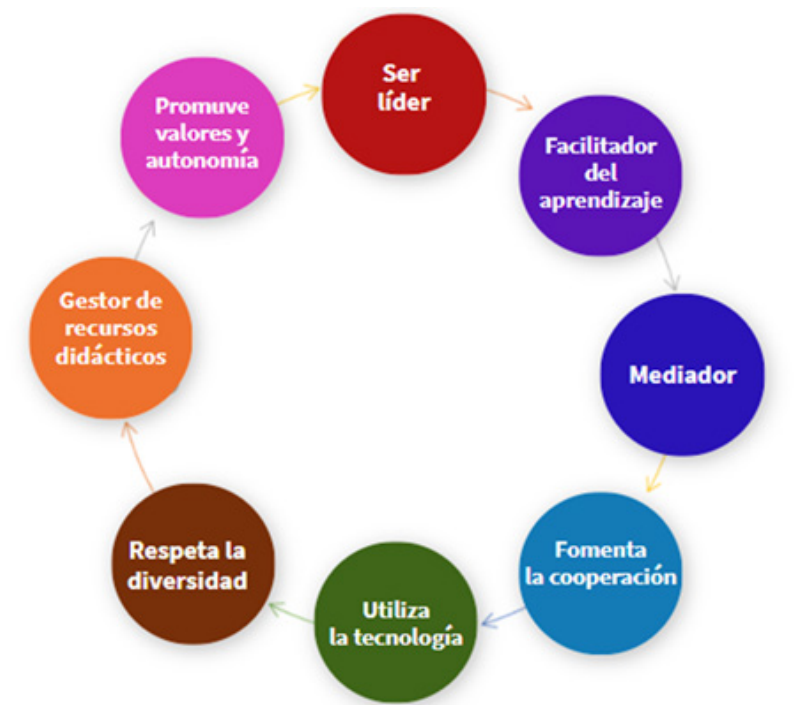

Figura 2. Habilidades docentes para los retos del presente. Fuente: Elaboración propia (2020).

Estas habilidades implican mostrar cómo el propio proceso educativo conlleva una serie de conocimientos, habilidades y actitudes necesarias para integrarlas al buen uso de la tecnología. A partir de la Pandemia Covid-19, el docente tuvo que sustentar sus clases en soportes digitales, diseñar recursos con aplicación de la tecnología, para romper con los paradigmas de la educación tradicional. Para ello, fue necesario considerar 
al estudiante como centro del proceso enseñanza-aprendizaje a través de los medios digitales, los contenidos interactivos y el uso de plataformas virtuales.

Asimismo, el saber hacer en la práctica educativa requiere del conocimiento de estrategias de aprendizaje, técnicas y saber cómo actuar con los objetos; significa romper esquemas de la educación tradicional para integrar los modelos actuales de aprendizaje para dar paso "a la construcción del conocimiento en los estudiantes como los métodos de casos, aprendizaje basado en investigación, aprendizaje basado en problemas, aprendizaje basado en proyectos, aprendizaje cooperativo, aula invertida, entre otras" (Rivadeneira, 2017, p.42). Es decir, el docente desarrolla la habilidad de hacer la clase dinámica y retadora a través de ambientes de aprendizaje virtual.

\section{Competencia digital docente}

La formación en competencia se basa en el reencuentro de dos corrientes teóricas de las ciencias de la educación: el cognitivismo y el constructivismo. Por un lado, el primero se ocupa de la manera en la que el docente adquiere y aplica los conocimientos y las habilidades; por otro lado, el segundo hace hincapié en el papel activo del docente. Por lo tanto, las competencias digitales implican el conocimiento básico sobre el funcionamiento de las TIC para generar ambientes de aprendizajes significativos. El docente actualizado en las competencias digitales debe ser capaz de "promover y ejemplificar la etiqueta digital y las interacciones sociales responsables relacionadas con el uso de las TIC y la información, y desarrollar y modelar la comprensión de diferentes culturas y la conciencia global" (Rangel, 2015, p.240), utilizando herramientas de comunicación y colaboración en el espacio virtual. La competencia digital docente requiere, por tanto, que el profesional de la educación diseñe y aplique recursos a través de herramientas digitales en línea, comparta recursos en la red y edite contenido virtual.

El docente al impartir clases en ambientes de aprendizaje virtuales, ya ha tenido que desarro- llar las habilidades digitales, con lo cual fomenta en los estudiantes el aprendizaje y los involucra en el desarrollo de competencias digitales. El docente a cargo de un espacio diseñado con la finalidad de llevar a cabo el proceso educativo, es decir, en un entorno virtual de aprendizaje, está obligado a diseñar, ejecutar y evaluar en la web. De igual manera, realiza actividades, exámenes $y$ trabajos colaborativos, sin tener que coincidir en un espacio y tiempo con sus estudiantes. De acuerdo con Ana Pereyra (2020), durante la Pandemia, la continuidad pedagógica se logró a través de plataformas educativas y computadoras conectadas a internet que permitió "la realización de tareas asincrónicas complementarias del trabajo presencial" (p.128).

Por otro lado, la propuesta de Marqués (2008) refiere que hay otras competencias de tipo informacional, las cuales son importantes que las docentes las conozcan y tengan acceso a las fuentes de información y recursos digitales, y que utilicen los programas informáticos relevantes y específicos de las materias que imparten (Rangel, 2015). En este sentido, el docente hace uso de la tecnología para fortalecer sus competencias digitales docentes, a la vez que fortalece el proceso de aprendizaje en los estudiantes, motivando en ellos un sentido crítico ante la información presente en el espacio virtual.

\section{Materiales y Métodos}

\section{Participantes}

La muestra estuvo conformada por 64 docentes de cuatro Instituciones de Nivel Medio Superior y Superior. Las edades de los docentes fueron entre 28 y 50 años de edad, de ambos sexos. La muestra fue no probabilística de una población de 375 docentes. De voluntad propia se escribieron al curso, mismos que laboran en el nivel de educación media y superior en la Universidad autónoma del Estado de Hidalgo campus Huejutla, Escuela Vocacional de Sistema de Educación Media Superior de la Universidad de Guadalajara, Colegio de Educación Estética y Artística de la Escuela 
Nacional Preparatoria (ENP), Universidad Azteca Plantel Acuña. La muestra significativa estuvo conformada por el $17.6 \%$ del universo en total.

\section{Instrumento}

El instrumento de recolección de datos fue una escala tipo Likert, integrada por 20 reactivos para identificar el nivel de competencias en lo que se refiere al diseño y producción de material didáctico con herramientas digitales. El proceso de validez de la escala se verificó mediante la opinión de cuatro expertos que asignaron una calificación promedio de $94 \%$ de aceptación de aplicabilidad del instrumento, (Hernández, Fernández y Baptista, 2010), acreditando de esta manera la aplicación del instrumento. La confiabilidad del instrumento se realizó a través de una prueba piloto, obteniendo una confiabilidad del Alpha de Cronbach de 0.89, con 20 ítems con escala de Likert con cinco opciones de respuestas (nada, poco, regular, bien y muy bien).

\section{Tipo y Diseño}

El presente estudio es descriptivo, de corte transversal y correlacional se utilizó el método cuantitativo, que permitió medir las competencias digitales de los docentes participantes, en lo que respecta al diseño y producción de material didáctico con el uso de herramientas digitales. Los datos se recogieron con base en una escala de medición numérica, para analizarlos mediante la estadística descriptiva a través de frecuencias.

\section{Procedimiento}

El estudio se realizó de la siguiente manera:

Etapa 1. Se realizó una revisión bibliográfica dentro del Estado de Arte. Lo cual ayudó a identificar los objetivos del estudio y elaborar el instrumento de análisis.

Etapa 2. Se diseñó la encuesta como herramienta de recolección de datos, y se gestionó la validación por parte de los expertos y la confiabilidad a través de un piloto.

Etapa 3. Aplicación del cuestionario, para lo cual se utilizó el formulario Google form para su recolección.

Etapa 4. Análisis de los resultados, la base de datos se obtuvo en formato Excel y el análisis estadístico se realizó en SPSS v. 21

\section{Confidencialidad y Consentimiento informado}

Los participantes se inscribieron de forma voluntaria para participar del estudio, se les informó sobre sus derechos y garantías como participantes en esta investigación. Habiendo ellos participado previamente en un curso taller del uso de las tecnologías de la información y comunicación, aceptando que se les aplique los instrumentos descritos para conocer el nivel de competencia digital que poseían. (APA, 2020).

\section{Resultados}

Para el análisis de los datos e interpretación de los resultados se presenta en la Figura 4, la frecuencia con la que los docentes participan y se comunican en entornos virtuales. La información arrojó que un 36\% de los docentes ocasionalmente o nunca participan y se comunican frecuentemente en entornos digitales.

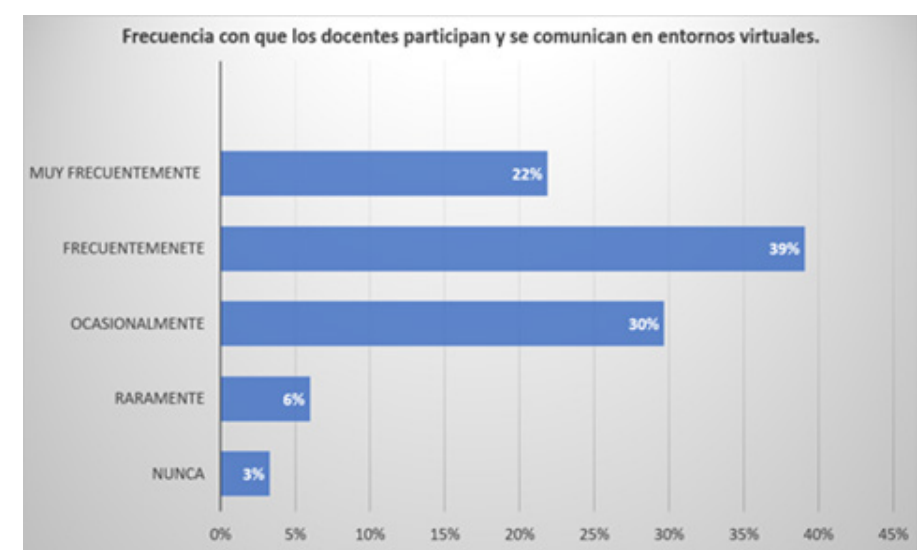

Figura 4. Frecuencia con que los docentes participan y se comunican en entornos virtuales.

Fuente: Elaboración propia (2021)

En la Figura 5, se puede observar que el 39\% de los docentes frecuentemente diseñan y aplican recursos a través de herramientas en línea. 


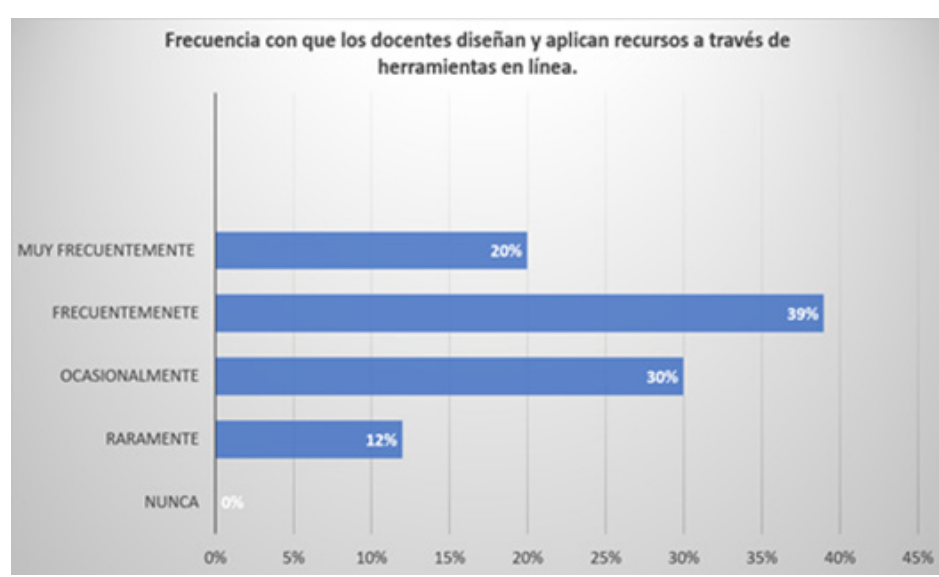

Figura 5. Frecuencia con que los docentes diseñan y aplican recursos a través de herramientas en línea.

Fuente: Elaboración propia (2021).

En la Figura 6 se muestra que el 46.9\% de los docentes encuestados ocasionalmente, raramente y/o nunca han creado o editado contenidos nuevos con herramientas digitales

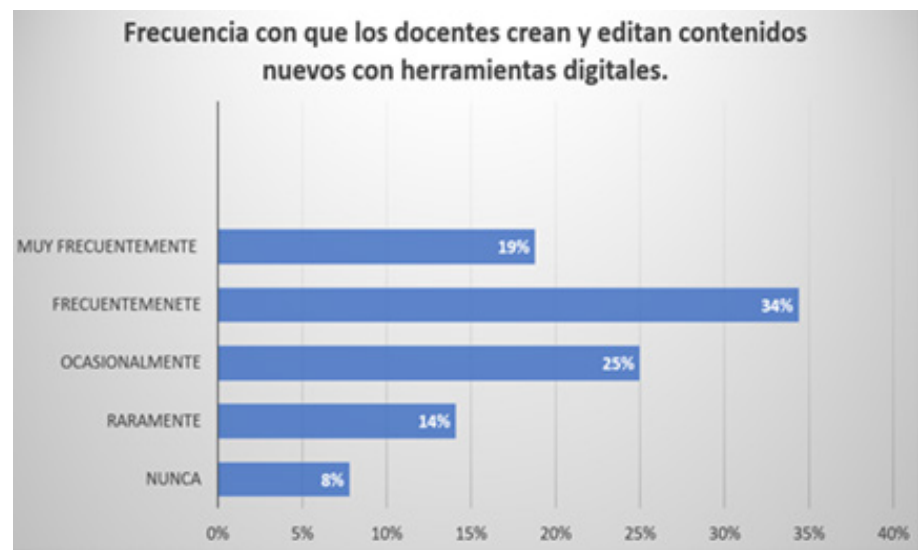

Figura 6. Frecuencia con que los docentes crean y editan contenidos nuevos con herramientas digitales. Fuente: Elaboración propia (2021).

La competencia digital docente mediante el uso de herramientas digitales se midió con dos criterios: 1) Frecuencia con que los docentes crean y editan contenidos nuevos con herramientas digitales, 2) Frecuencia en que los docentes participan y se comunican en entornos virtuales. La Tabla 1, muestra que el $34.4 \%$ de los docentes frecuentemente crean contenidos con herramientas digitales. Mientras que sólo $7.8 \%$ nunca crea y edita contenidos nuevos con herramientas digitales.
Tabla 3. Frecuencia con que los docentes crean y editan contenidos nuevos con herramientas digitales.

\begin{tabular}{|c|c|c|}
\hline & \multicolumn{2}{|c|}{ Uso de herramientas digitales } \\
\hline & $\mathrm{F}$ & $\%$ \\
\hline Nunca & 3 & 7.8 \\
\hline Raramente & 5 & 14.1 \\
\hline Ocasionalmente & 8 & 25 \\
\hline Frecuentemente & 12 & 34.4 \\
\hline $\begin{array}{l}\text { Muy } \\
\text { frecuentemente }\end{array}$ & 6 & 18.8 \\
\hline Total & 34 & 100 \\
\hline
\end{tabular}

Fuente: Elaboración propia (2021)

El componente de usos de la tecnología en aulas no presenciales, medida con la frecuencia con la que los docentes participan y se comunican en entornos virtuales. Los resultados de la Tabla 4 muestran que la mayoría de los docentes encuestados responde que frecuentemente participan y se comunican en entornos digitales (39.1\%) y el $3.3 \%$ responde que nunca.

Tabla 10. Frecuencia con que los docentes participan y se comunican en entornos digitales.

\begin{tabular}{lrr}
\hline \multicolumn{3}{c}{ Uso de herramientas digitales } \\
\hline Nunca & $\mathrm{F}$ & $\%$ \\
Raramente & 1 & 3.3 \\
Ocasionalmente & 2 & 6 \\
Frecuentemente & 10 & 29.7 \\
Muy & 14 & 39.1 \\
frecuentemente & 7 & 21.9 \\
\hline Total & & \\
\hline
\end{tabular}

Fuente: Elaboración propia (2021)

\section{Discusión y Conclusiones}

El estudio nos muestra que el $46.9 \%$ de los docentes encuestados ocasionalmente, raramente y/o nunca han creado o editado contenidos nuevos con herramientas digitales, lo cual asevera lo indicado por (Magro, 2020) al referir que la pandemia ha mostrado a todos las desigualdades educativas existentes. Esto se debe, en gran medida, a que no todos los docentes han desarrollado habilidades para transmitir conocimientos y fomentar destrezas relacionadas con las herramientas digitales. Por lo que los docentes deben 
tener claro lo que menciona Dussel, Ferrante y Pulfer (2020, p. 364) "...la sola introducción de equipamiento tecnológico, sea en la opción que sea (notebook, plataformas, apps o pizarra digital interactiva), produciría un salto cualitativo automático en los procesos de formación."

Urge un fomento al desarrollo de la formación docente en el uso de herramientas digitales y escenarios virtuales para generar el diálogo entre estudiantes y docentes. Los resultados encontrados de poca o nula participación en entornos virtuales por parte de los docentes, demostró inequidad en el acceso a las TIC, como lo señalan Mancera, Serna y Barrios (2020). Y como lo refiere Schleider \& Reimers, (2020) que esta situación de pandemia evidenció la urgente necesidad de manejar los entornos virtuales por parte de los docentes.

Los resultados encontrados en este estudio nos llevan a la reflexión en tiempos en el que la educación se ha virtualizado y como manifiesta la UNESCO (2019), los "sistemas educativos deben actualizar y mejorar regularmente la preparación y la formación profesional del personal docente y velar por que todos los profesores puedan sacar partido de la tecnología con fines educativos". Y como también lo refiere Alvites-Huamaní (2017) que las TIC son recursos y herramientas tecnológicas que facilitan el quehacer del docente y a su vez permite el desarrollo de distintas habilidades, destrezas y competencias de los estudiantes al mejorar la cognición y el proceso de aprendizaje.

El estudio deja abierta otras líneas de investigación, como es el seguimiento a los docentes que participaron y cómo implementan las herramientas aprendidas en su práctica diaria, además de estudiar la resistencia al cambio en aquellos que siguen utilizando una metodología tradicional a través de las TIC.

\section{Referencias Bibliográficas}

Álvarez, P. R., González Alfonso, M. C., y López Aguilar, D. (2009). La enseñanza universitaria y la formación para el trabajo: un análisis desde la opinión de los estudiantes. Paradigma,
30(2), 7-20. http://ve.scielo.org/scielo.php?pi$\mathrm{d}=$ S1011-22512009000200002\&script=sci_arttext\&tlng=en

Aguaded, J. I., Marínz, I., \& Díaz, E. M. (2015). La alfabetización mediática entre estudiantes de primaria y secundaria en Andalucía (España). RIED: Revista Iberoamericana de Educación a Distancia, 18(2), 275-298.

https://doi.org/10.5944/ried.18.2.13407

Alvites-Huamaní, C. (2017) Herramientas TIC en el aprendizaje en el área de Matemática: Caso Escuela PopUp, Piura-Perú. Hamut'ay, 4 (1), 1830. https://doi.org/10.21503/hamu.v4i1.1393

Argudín, Y. (2001). Educación basada en competencias. Nociones y antecedentes. México: Editorial Trillas.

Artismuño, A. (2005). Las competencias en la educación superior: ¿demonio u oportunidad? Montevideo. Universidad Católica del Uruguay, Departamento de Educación. http://www.upf. edu/bolonya/butlletins/2005/febrer1/demonio. pdf

APA - American Psycgological Association (2010). Manual de Publicaciones de la American Psychological Association.

(3ra. Ed.) México: El Manual Moderno.

Barnett, R. (2001). Los límites de la competencia: el conocimiento, la educación superior y la sociedad. España: Editorial Gedisa.

Chan, M. E., y Delgado, L. (2009). Principios de complejidad aplicados al diseño curricular por competencias. Ponencia presentada en el V Congreso Internacional sobre el Enfoque Basado en Competencias. Bogotá, CO: CIEBC.

Dussel, I; Ferrante, P. y Pulfer, D. (2020). Nuevas ecuaciones entre educación, sociedad, tecnología y Estado. Dussel, I; Ferrante, P.; Pulfer, D. (compiladores). Pensar la educación en tiempos de pandemia. Entre la emergencia, el compromiso y la espera. Buenos Aires, Argentina: UNIPE-Editorial Universitaria, pp. 351-364.

Hernández, R; Fernández, C. y Baptista, P. (2010). Metodología de la Investigación. (5ta. Edición). México, D.F: McGraw Hill.

Hernández, R., Fernández, C., \& Baptista, M. P. (2014). Metodología de la investigación. Madrid: McGraw Hill. 
Hodges, C., Moore, S., Lockee, B., Trust, T., y Bond, A. (2020). La diferencia entre la enseñanza remota de emergencia y el aprendizaje en línea. Educause Review. https://er.educause.edu/ articles/2020/3/the-difference-betweenemergency-remote-teaching-and-onlinelearning.

Ibáñez, F. (2020). Educación en línea, virtual, a distancia y remota a distancia. Observatorio de Innovación Educativa. https://observatorio.tec. $\mathrm{mx} / \mathrm{edu}$-news/diferencias-educacion-online-virtual-a-distancia-remota

Justo, S. \& Quintanilla, G. (2020). Mi inicio en la docencia. Comisión Nacional para la Mejora Continua de la Educación. México.

Limón, C. (2020). Tecnología y habilidades para sistemas educativos en un mundo en evolución continua. En Granados Roldán, O. (2020). La educación del mañana. ¿Inercia o transformación? (pp. 75-82). Madrid, España: Organización de Estados Iberoamericanos para la Educación, la Ciencia y la Cultura (OEI).

OCDE (2019). "Teachers and School Leaders as Lifelong Learners". In Education at a Glance 2019: OECD Indicators OECD Publishing, Paris. Mancera, C., Serna, L. y Barrios, M. (29 de abril 2020). Pandemia: maestros, tecnología y desigualdad. Nexos, blog de educación: https://educacion.nexos.com. $\mathrm{mx} / \mathrm{p} \mathrm{p}=2286$

Magro, C. (2020). Esta crisis ha mostrado la fragilidad del sistema educativo. Revista Educación 3.0, 17 de septiembre de 2020. https://www.educaciontrespuntocero.com/entrevistas/carlos-magro-crisis-sistema-educativo/

Mejoredu (2020). Comisión Nacional para la Mejora Continua de la Educación. La política social en el contexto de la pandemia por el virus SARS-CoV-2 (COVID-19) en México. https:// www.coneval.org.mx/Evaluacion/IEPSM/Documents/Politica_Social_COVID-19.pdf

Naffi, N. (2020). Disruption in and by Centres for Teaching and Learning During the COVID-19 Pandemic: Leading the Future of Higher: LOObservatoire Internationale sur les Impacts Sociétaux de l'IA et du Numerique and the Government of Québec, https://cutt.ly/6fQZibh.

Pereyra, A. (2020). Viejas y nuevas desigualdades educativas. Desafíos emergentes a la formación docente. Dussel, I.; Ferrante, P. (compliladores). (2020). Pensar la educación en tiempos de Pandemia. Entre la emergencia, el compromiso y la espera. Buenos Aires, Argentina: UNIPE, Editorial Universitaria, pp. 125-136.

Reich, J. (2020). Failure to Disrupt. Why Technology Alone Can't Transform Education. Harvard University Press. https://doi. org/10.4159/9780674249684

Rangel, A. (2015). Competencias docentes digitales: propuesta de un perfil Pixel-Bit. Revista de Medios y Educación. Sevilla, España: Universidad de Sevilla, 46 (1), 235-248. https://doi. org/10.12795/pixelbit.2015.i46.15

Rivadeneira Rodríguez, E. M. (Julio, 2017). Consultado en Competencias didácticas-pedagógicas del docente, en la transformación del estudiante universitario. Revista Científica Ciencias Humanas. Venezuela: Orbis. vol. 13, núm. 37, julio, 2017, pp. 41-55. https://www.redalyc.org/ pdf/709/70952383003.pdf

Rodríguez, N. (2011). Diseños experimentales en educación. Revista de Pedagogía, 32(91), 147-158.

Schleicher, A., \& F. Reimers (2020), Schooling Disrupted, Schooling Rethought: How the COVID-19 Pandemic is Changing Education, OECD, https://read.oecd-ilibrary.org/view/?re$\mathrm{f}=133 \_133390$-1rtuknc0hi\&title=Schooling-disruptedschooling-rethought-How-the-Covid-19-pandemic-is-changing-education.

UNESCO. (2019). Marco de Competencias de los Docentes en materia de TIC. Organización de las Naciones Unidas para la Educación, la Ciencia y la Cultura. https://es.unesco.org/themes/ tic-educacion/marco-competencias-docentes. 